\title{
Briefwechsel mit Führer und Co. Texte aus Bruno Thürings Zeit an der Universitätssternwarte Wien
}

\author{
Franz Kerschbaum \\ Institut für Astronomie der Universität Wien, \\ Türkenschanzstraße 17, A-1180 Wien, Austria
}

\begin{abstract}
Bruno Thüring (1905-1989) acted as director of Vienna's University Observatory from 1941-45. He was one of the multitude of scientists joining the campaign against Einstein's Theory of Relativity which was undertaken in the name of so-called "German Physics" (Deutsche Physik) between the 1920s and the 1940s. Related letters available in local Viennese University archives document his contacts with a number of at that time important people in the field of science politics and allow for some eye opening insights into privat and behind the scenes networking.
\end{abstract}

\section{Zeitrahmen}

Von 1941-1945 war Bruno Thüring Direktor der Universitätssternwarte Wien. Der Mitstreiter für die Deutsche Physik stand im Kontakt mit wissenschaftspolitisch wichtigen Vertretern des Deutschen Reiches und hat einen reichen Fundus an offiziellen und halboffiziellen Schriftstücken in Wien zurückgelassen.

Bevor auf individuelle Briefe aus dem Umfeld Bruno Thürings eingegangen wird, soll kurz das zeitliche Umfeld an der Wiener Universitätssternwarte skizziert ${ }^{1}$ werden.

Während der 30er Jahre sind die beiden Professuren mit Kasimir Graff (Praktische Astronomie, zugleich Direktor) sowie Adalbert Prey (Theoretische Astronomie) besetzt. Schon knapp vor der Emeritierung stehend, wird Graff

\footnotetext{
${ }^{1}$ Eine ausführliche Darstellung des Direktorats Thüring findet sich in: F. Kerschbaum, T. Posch, K. Lackner, Die Wiener Universitätssternwarte unter Bruno Thüring, in: Beiträge zur Astronomiegeschichte, Band 8, hg. von W.R. Dick und J. Hamel, S. 185-202, Frankfurt a.M. 2006
} 


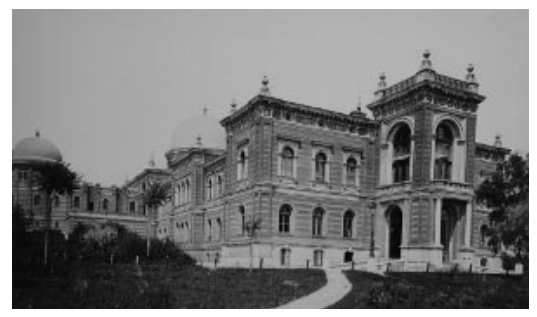

Abb. 1: Die Wiener Universitätssternwarte im frühen 20. Jahrhundert.

unmittelbar nach dem Anschluss Österreichs an das Deutsche Reich mit dem formalen Vorwurf der Unterschlagung beurlaubt und Prey als kommisarischer Leiter eingesetzt. Graff wird kein schuldhaftes Verhalten nachgewiesen und nach dem ihm nahegelegten freiwilligen Ausscheiden die finanziell wichtige „,Entpflichtung" ermöglicht.

Prey, auch vor der Emeritierung stehend, arbeitet für beide Lehrkanzeln Nachfolgevorschläge aus, die aber vom zuständigen Beamten im Reichserziehungsministerium, Wilhelm Führer unbeachtet bleiben. Der spätere Obersturmführer der Waffen-SS, ursprünglich ausgebildeter Astronom, in weiterer Folge Dozentenbundführer, ist stark wissenschaftspolitisch tätig und hat eigene Pläne mit Wien.

Seit 1933 mit Bruno Thüring befreundet, besetzt er die Lehrstühle mit Thüring und Karl Schütte. Bruno Thüring hat sich in den Jahren davor als engagierter Vertreter der ,,Deutschen Physik“ und polemischer EinsteinKritiker $^{2}$ einen einschlägigen Ruf erarbeitet. Der Dienstantritt Thürings erfolgt am 20. Jänner 1941; ab 15. März 1943 bis Kriegsende steht Thüring aber im (ungeliebten) Wehrdienst. Karl Schütte wird schon nach seiner Antrittsvorlesung eingezogen und nie in Wien tätig. Nach Kriegsende erfolgen im März 1945 letzte Zahlungen an Thüring und Schütte. Beide werden mit 23. August des Jahres aus dem Personalstand der Universität Wien gelöscht.

Kasimir Graff, aus der Emeritierung ,,zurückgeholt“, leitet die Sternwarte wieder bis Oktober 1948. Der bei Kriegsende fluchtartig sich in den Westen absetzende Thüring besucht erst im Mai 1946 Wien, um Privateigentum (nur teilweise erfolgreich) zu übersiedeln. In weiterer Folge stuft die Bayrische Spruchkammer Bruno Thüring zuerst 1949 als ,,minderbelastet “, 1950 als ,,Mitläufer “ ein. Josef Hopmann folgt 1951 Graff in Professur und Leitung der Sternwarte.

Die Theorie-Professur Preys wird 1954 mit Konradin Ferrari d'Occhieppo nachbesetzt. Bruno Thüring stirbt am 6. Mai 1989 in Karlsruhe.

\footnotetext{
${ }^{2}$ Eine umfassende Diskussion dazu findet sich in: Bruno Thürings ,,philosophische “ Kritik an Albert Einsteins Relativitätstheorie, T. Posch, F. Kerschbaum, K. Lackner, Wiener Jahrbuch für Philosophie, Bd. 38 (2006), Wien 2007, p. 269-291
} 


\section{Dokumentenbestand, Quellen}

Die Primärquellenlage zur Zeit um die Direktion Thüring ist als sehr gut zu bezeichnen. Einerseits konnte direkt auf die an der Universitäts-Sternwarte vorhandenen Personalakte von Thüring bzw. Graff zurückgegriffen werden. Es finden sich darin knapp 100 Schriftstücke aus den Jahren 1938 bis 1951, darunter Notizen, Eingaben, offizielle und halboffizelle Briefe inklusive Abschriften von abgegangenen Schriftstücken. Das ganze Material wurde elektronisch erfasst und liegt digital in Volltextform vor.
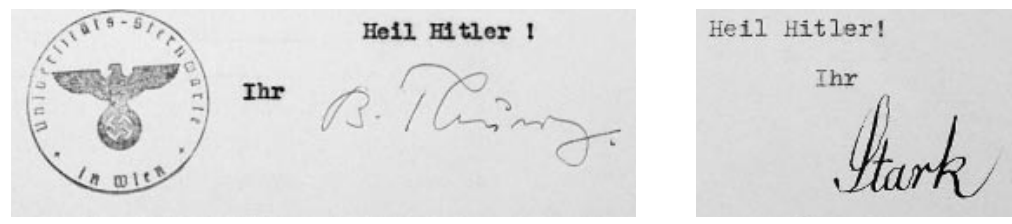

Abb. 2: Links: Signatur Thürings aus einem Schreiben vom 19. 3. 1942 an Paul Guthnik - Rechts: Signatur des Gratulationsschreiben vom 22. 12. 1940 von Johannes Stark anlässlich der Berufung Thürings nach Wien.

Andererseits bot auch das Universitätsarchiv mit seinen Akten der damaligen philosophischen Fakultät der Universität Wien eine Vielzahl von interessanten Schriftstücken. Alle mit dem Gegenstand unserer Arbeit im Zusammenhang stehenden wurden ebenso zumindest digital abfotografiert, um für weitere Nutzung einfacher zur Verfügung zu stehen. Für die Zukunft ist geplant, die Materialien auch elektronisch zu publizieren.

Als typische Beispiele sollen in den beiden folgenden Abschnitten nun einzelne Schriftstücke ${ }^{3}$ kurz vorgestellt werden.

\section{,Hohe" Politik}

Die Freundschaft mit Wilhelm Führer und der daraus folgende Briefwechsel lässt viele Facetten der NS-Wissenschaftspolitik erahnen. Schon in der oben angesprochenen Publikation Die Wiener Universitätssternwarte unter Bruno Thüring haben wir einen sehr aufschlussreichen Privatbrief von Führer an Thüring veröffentlicht, der bereits Mitte 1939 all seine Pläne für die Neuordnung der astronomischen Landschaft im Dritten Reich aber auch viele andere Details

\footnotetext{
${ }^{3}$ Weitere Beispiele finden sich bereits publiziert in: F. Kerschbaum, T. Posch, K. Lackner, Die Wiener Universitätssternwarte unter Bruno Thüring, in: Beiträge zur Astronomiegeschichte, Band 8, hg. von W.R. Dick und J. Hamel, S. 185-202, Frankfurt a.M. 2006
} 


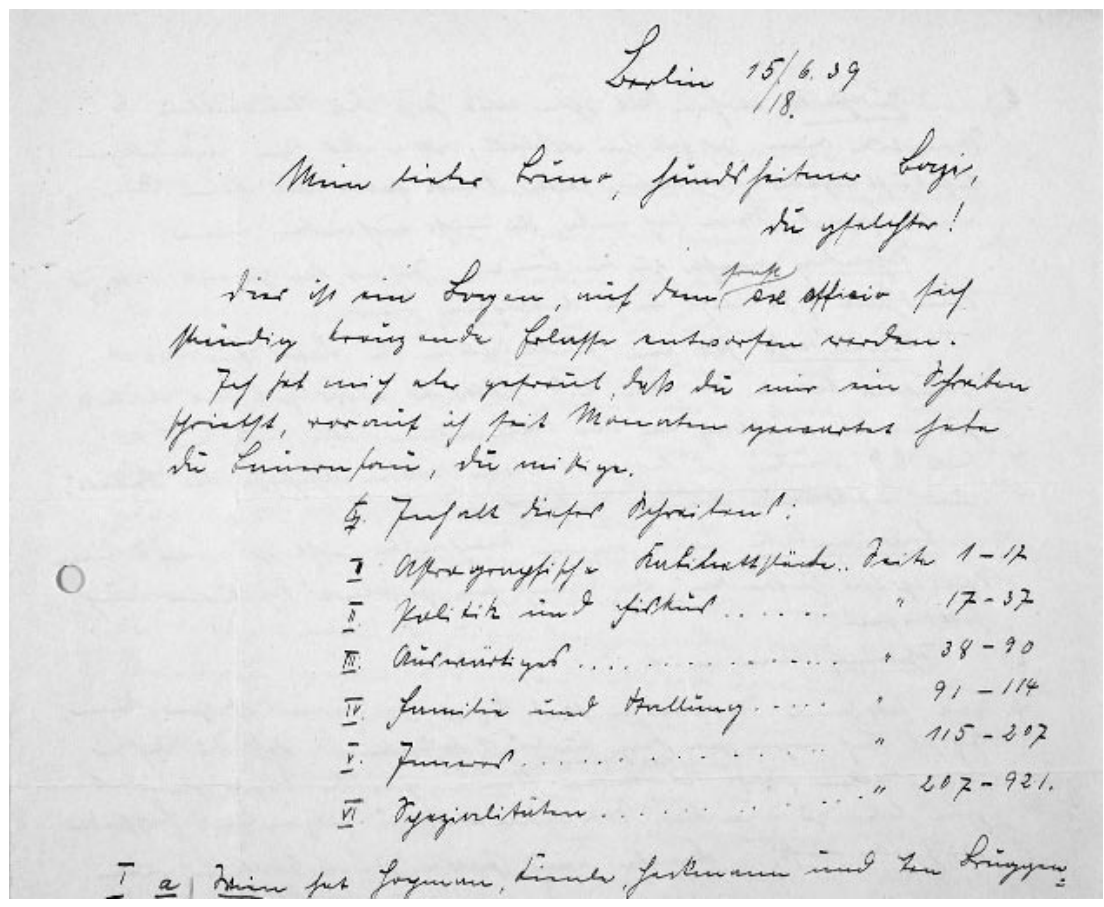

Abb. 3: Ausschnitt aus einem Privatbrief von Führer an Thüring vom Juni 1939

seiner Arbeit offenbart. Lokalere Beispiele zu Besetzungsfragen offenbaren sich im Gratulationsbrief von Johannes Stark an Thüring vom 22.12.1940:

[...] Es wäre sehr erfreulich, wenn Sie sich in Wien auch um die Physik etwas annehmen würden. Mit ihr steht es dort nicht erfreulich, da Heisenberg für die theoretische Professur vorgeschlagen werden sollte $[. .$.

oder dem sehr privaten und zotig verfassten Brief Thürings an Führer vom 7. 2. 1942:

[...] Ueber den Verlauf der Sitzung über den Lehrstuhl für Geschichte und Methodik wirst Du bereits vom Dekan informiert worden sein und es verbleibt mir nur noch die Aufgabe, ergänzend und wohl auch deutlicher zu berichten. [...] Endergebnis: Lehrstuhl sowie Kandidat [Dingler] wurden mit 
n-1 Stimmen (wo $n$ die Anzahl der Teilnehmer ist) abgelehnt. Die eine Stimme war ich. [...] Die Argumente gegen die Kandidatur Dingler bestanden in verzerrten Darstellungen dessen, was er angeblich in seinen Büchern lehre und in seinen Wiener Vorlesungen gesagt habe. Die Verzerrungen waren teilweise derart lächerlich, dass es mir schwer wurde, nur an Missverständnisse zu glauben. [...] Die Geistesverhärtung der Naturwissenschaftler von heute ist mir wieder einmal grauenhaft deutlich geworden. Es vermischt sich da Denkfaulheit mit Denkunvermögen zu einem unentwirrbaren Knäuel. [...]

Letzteres Schreiben zeigt, dass Thüring, ganz im Sinne von Starks Wünschen im vorhergehenden Beispiel, versucht hat, in Wien Besetzungen zu beeinflussen. Er war dabei aber augenscheinlich nicht sehr erfolgreich, und dies nicht nur beim Versuch, Hugo Dingler nach Wien zu bekommen. Thüring hat auch verschiedentlich für ihm nahe stehende Personen interveniert, wie im Brief an Paul Guthnick vom 19. 3. 1942:

[...] Prof.Dr. Schütte, der ja gegenwärtig an der Deutschen Seewarte in Hamburg tätig ist, hat sich vor einiger Zeit mit der Bitte an mich gewandt, bei Ihnen als dem Vorsitzenden der $A D A^{4}$ anzuregen, auch ihn in den ADA aufzunehmen unter Berücksichtigungen des Umstandes, dass er einen selbständigen astronomischen Lehrstuhl (in Wien) innehat und in jüngerer Zeit auch Astronomen in den ADA aufgenommen worden sind (z.B.Prof. Stumpff, Prof. Unsöld), die nicht gerade Direktoren von Sternwarten sind. [...]

oder versucht Fördermaßnahmen zu beeinflussen, wie im Schreiben an Führer vom 24. 5. 1942:

[...] Im Börsenblatt vom 9. April kündigen Pasqual Jordan und der Holmst Meyer-Abich die Herausgabe einer neugegründeten Zeitschrift an: "Physics" Beiträge zur naturwissenschaftlichen Synthese. Dafür ist also mitten im Kriege Papier da. Ich lege statt langatmiger Erörterungen einen Brief Mays an Kisshauer bei. Ich bitte Dich, zu erwägen, ob nicht die Möglichkeit besteht, von Seiten des REM aus in letzter Minute gegen diese unnötige und schädliche Neugründung Sturm zu laufen und sie zu verhindern. [...]

\footnotetext{
${ }^{4}$ Dem Ausschuss der Deutschen Astronomen ADA gehörten damals die Leiter aller selbstständigen Deutschen Sternwarten an.
} 
Thürings explizit antisemitische Reiseberichte und Meldungen an Führer sind schon in Die Wiener Universitätssternwarte unter Bruno Thüring auszugsweise zitiert worden.

Diese Beispiele sollen Bruno Thürings Verflechtungen illustrieren. Fast immer liefen seine Aktivitäten aber über Wilhelm Führer. Nach dessen Abgang aus dem Reichserziehungsministerium ab 1943 - Führer war im Weiteren im Amt Ahnenerbe und als Ordonanzoffizier im persönlichen Stab des Reichsführers SS (Himmler) tätig — hatte Thüring nicht mehr diesen Kontakt zu den Schaltstellen der Wissenschaftspolitik.

\section{Das lokale Leben}

Die politischen Brüche und die wirtschaftlichen Schwierigkeiten spiegeln sich auch in den mehr lokalen bzw. privaten Bedürfnissen wider. So wurde längere Zeit um die Benutzung der Dienstwohnung, aber auch der wissenschaftlichen Einrichtungen zwischen Graff und Thüring teilweise mit dem zwischengeschalteten Prey gerungen - und dies im Laufe der Jahre aus sehr unterschiedlichen Verhandlungspositionen. So schreibt der recht schnell an die neuen Verhältnisse angepasste Adalbert Prey am 16. 9. 1939 vertraulich an den Rektor:

\section{[...] Durch seine Zuweisungsverfügung des Standortkommandos [...] vom 10.7.1939 ist die Sternwarte für die Deutsche Seewarte sichergestellt worden. Daraus folgt, dass sie bis auf weiteres für alle Besuche gesperrt ist. In diesem Zusammenhange stellt die unterzeichnete kommissarische Leitung die Frage, ob man dem ehemaligen Direktor der Sternwarte, Dr. K. Graff, der mit dem Umbruch beurlaubt wurde, auch weiter noch den Zugang zu den Instrumenten gestatten soll. [...]}

um dann schon am 28. 9. 1939 Graff zu informieren:

[...] Auf Ihre Anfrage vom 26.d.M. bezüglich der Benutzung des Grossen Refraktors muss ich Ihnen leider mitteilen, dass ich diese vorläufig nicht gestatten kann. Dagegen kann gegen Beobachtungen in der Hütte oberhalb Ihrer Wohnung nichts eingewendet werden und es steht Ihnen auch der Mechaniker zur Aufstellung Ihres [privaten] 6-Zöllers zur Verfügung. [...]

Graff war zu diesem Zeitpunkt nicht emeritiert, sondern nur beurlaubt und sollte offensichtlich an weiteren Arbeiten mit dem Großen Refraktor gehindert werden. Kurz nach dem Eintreffen Thürings in Wien 1940/41 war das Dienstwohnungsproblem zu lösen. Graff, ohne Schuldbeweis und angesichts 
der schwieriger werdenden Wohnungssituation in Wien, war nicht willens, die Direktionswohnung für Thüring zu räumen, worauf sich dieser am 2. 5. 1941 beim zuständigen Kurator der Universität beschwert:

[...] Zu all dem kommt, dass Prof. Graff keine besondere persönliche Rücksichtnahme in der zur Debatte stehenden Hinsicht verdient, da er aus politischen Gründen aus seinem Amt ausschied. Aus dem gleichen Grunde konnte ja auch schon, wie mir im Kuratorium mitgeteilt wurde, der Weg zur Wohnungsvermittlung durch stellvertretenden Gauleiter Scherizer gar nicht beschritten werden. Ich kann aber nicht einsehen, dass meine Arbeitsmöglichkeit vermindert werden soll nur wegen der politischen Mangelhaftigkeit Prof. Graffs und seinen durch keinerlei dienstliche und lebenswichtige Umstände begründbaren Wunsch, ausgerechnet in Wien wohnen zu wollen. [...]

Bemerkenswert ist hier das ,,aus politischen Gründen”. In allen anderen offiziellen Dokumenten zur Causa Graff geht es ja immer um die ihm vorgeworfenen Unterschlagungen und Unregelmäßigkeiten, die ihm trotz aller Mühe nicht nachgewiesen werden konnten. Von einer Räumungsklage bedroht, zieht Graff am 21. 6. 1941 aus.

Das ,Wohnungsspiel" dreht sich nach Kriegsende wieder in die andere Richtung. Schon am 2. 6. 1945 zieht Graff in ,,seine" Dienstwohnung ein und listet penibel alle Einrichtungsgegenstände Thürings auf ${ }^{5}$. Er begründet dies in einem Schreiben an die Staatsgebäudeverwaltung vom 10. 4. 1946:

[...] Wegen der Verluste, die ich durch den seinerzeitigen übereilten Umzug (Räumungsklage ohne Wohnungszuweisung) und durch die Kriegsereignisse (Brand- und Wasserschaden, Plünderung anlässlich der drei Monate dauernden militärischen Hausbesetzung) erlitten habe, war ich gezwungen, die von meinem Vorgänger Bruno Thüring bei seiner Flucht aus Wien zurückgelassenen Möbel in Benutzung zu nehmen, in der Hoffnung, im Laufe des Jahres den grössten Teil derselben durch eigene Neuerwerbung ersetzen zu können. Da dies sich bisher als völlig unmöglich erwiesen hat, bitte ich um die Genehmigung, wie bisher die notwendigsten Hausgegenstände weiter benutzen zu dürfen. Der Ordnung halber lege ich aber eine Liste des Inventars der Thüringschen Wohnung bei, wie ich es im Juni vorigen Jahres hier vorgefunden habe, und das als Grundlage für die spätere Uebernahme der Gesamteinrichtung durch die Behörde

\footnotetext{
${ }^{5}$ Vom ,,Totenkopf”-Ölbild bis zum Sahnekännchen mit Sprung
} 
dienen soll. Professor Th ür ing war Mitglied der N.S.D.A.P. und seit dem 1. Jänner 1941 S.A.-Obertruppführer. [...]

Wie schon oben notiert, holt sich Thüring im Mai des Jahres seine restlichen privaten Dinge ab. Nicht ausgefolgt wird ihm aber jener Teil seiner Privatbibliothek, der sich aus eindeutig belasteten Druckwerken von Autoren wie Chamberlain, Dwinger, Grunsky, Günther, Hitler und Ludendorff zusammensetzt.

\section{Ausblick}

Die in dieser und früheren Arbeiten gegebenen Einblicke in das reiche archivarische Material um die Direktionszeit Bruno Thürings zeigen drei klare Linien zukünftiger Arbeiten auf: (1) die systematisierte Zugänglichmachung in elektronischer Volltextform; nur so kann das umfangreiche Material in interdisziplinärer Form gemeinsam aufgearbeitet werden, (2) Bearbeitung einzelner besonders interessanter Schriftstücke, insb. auch auf fachastronomische Aspekte hin, und (3) die Erweiterung des Blicks auf die Nachbardisziplinen der Natur- und Formalwissenschaften an der Universität Wien. Auch hier sind schon erste Kontakte zu vergleichbaren Aktivitäten geknüpft.

\section{Literatur}

Kerschbaum, F., Posch, T., Lackner, K., 2006, Die Wiener Universitäts-Sternwarte unter Bruno Thüring, in: Beiträge zur Astronomiegeschichte, Band 8, hg. von W. R. Dick und J. Hamel, S. 185-202, Harri Deutsch Verlag, Frankfurt a.M.

Posch, T., Kerschbaum, F., Lackner, K., 2006, Bruno Thürings "philosophische" Kritik an Albert Einsteins Relativitätstheorie in: Wiener Jahrbuch für Philosophie, Bd. 38, Wien 2007, p. $269-291$

\section{Danksagung}

Besonderer Dank gilt Jeannette Höfinger und Marion Solar im Zusammenhang mit der Archivarbeit sowie Thomas Posch für wertvolle Diskussionen und Anregungen. 